\title{
Tunable Tsallis Distributions in Dissipative Optical Lattices
}

\author{
P. Douglas, S. Bergamini, and F. Renzoni \\ Department of Physics and Astronomy, University College London, Gower Street, London WC1E 6BT, United Kingdom
}

(Received 10 January 2006; published 24 March 2006)

\begin{abstract}
We demonstrated experimentally that the momentum distribution of cold atoms in dissipative optical lattices is a Tsallis distribution. The parameters of the distribution can be continuously varied by changing the parameters of the optical potential. In particular, by changing the depth of the optical lattice, it is possible to change the momentum distribution from Gaussian, at deep potentials, to a power-law tail distribution at shallow optical potentials.
\end{abstract}

DOI: 10.1103/PhysRevLett.96.110601

PACS numbers: 05.40.Fb, 05.60.Cd, 32.80.Pj

Statistical physics deals with systems composed of a large number of particles. The state of such systems is usually described by a distribution function, which allows us to determine the relevance of a certain configuration and to calculate macroscopic quantities, as the mean of physical observables. Gaussian distributions often occur whenever dealing with systems consisting of a large number of particles. These distributions well describe dynamics dominated by a large number of small random events, as, for example, the erratic motion of a small particle in water (Brownian motion). Not every system, however, can be described by Gaussian distributions, and there are situations in which the dynamics is dominated by rare and large fluctuations, in striking contrast with the Brownian motion corresponding to a Gaussian distribution. These large fluctuations result in long, power-law tail distributions, commonly termed Lévy distributions [1]. Associated to the long tail is the divergence of the first and/or second moment of these distributions.

Quite recently, power-law tail distributions have been attracting much attention, as many different systems, from atoms undergoing subrecoil cooling [2] to blinking nanocrystals [3], were found to follow Lévy statistics. Among the various systems governed by non-Gaussian statistics, cold atoms in optical lattice [4] attracted particular attention because of their tunability. It was, in fact, theoretically shown that by changing the lattice's parameters it should be possible to observe the transition between Gaussian and power-law tail distributions $[5,6]$. The experimental observation of anomalous fluctuations in the energy of an atom in an optical lattice constituted clear evidence of the predicted anomalous dynamics [7]. Further theoretical work [8] pointed out that the atomic momentum distribution is precisely a Tsallis distribution [9], a particular power-law tail distribution introduced in the context of nonextensive statistical mechanics. It was shown, furthermore, that the transition between Gaussian and power-law tail distributions is accompanied by a transition from ergodic to nonergodic dynamics [10]. In the present work, we demonstrate experimentally that the momentum distribution of cold atoms in an optical lattice is indeed a Tsallis distribu- tion, whose parameters can be continuously varied by changing the lattice's parameters.

Before presenting our experimental results, it is important to set the theoretical background. For simplicity, we restrict our theoretical analysis to the case of a $J_{g}=1 / 2 \rightarrow$ $J_{e}=3 / 2$ atom illuminated by two counterpropagating and orthogonally polarized light fields (the so-called lin $\perp$ lin configuration). The interaction with the laser fields produces a periodic potential $U_{ \pm}=U_{0}[-2 \pm \cos 2 k z] / 2$ for each ground state $| \pm\rangle$, where $z$ is the atomic position along the axis $O z$ of light propagation. Light-induced stochastic transitions between the two optical potentials lead to a damping mechanism, the so-called Sisyphus cooling, and to fluctuations in the atomic dynamics [4]. The damping force is momentum dependent, of the form $F=-\alpha p$ / $\left[1+\left(p / p_{c}\right)^{2}\right]$, where $\alpha$ is constant, $p$ is the atomic momentum, and $p_{c}$ is a characteristic capture momentum, which depends on the lattice parameters [11]. The finiteness of the capture momentum results in a power-law tail momentum distribution, with sufficiently energetic atoms undergoing long flights over many potential wells [5,6].

A precise analysis of the atomic momentum distribution was carried out in the framework of the Fokker-Planck equation (FPE) for the atomic semiclassical Wigner function [11]. An analytic solution of the FPE was obtained in the limit of negligible spatial modulation, i.e., after spatial averaging over a period of the optical potential. It was later pointed out [8] that the solution of the FPE determined in Ref. [11] is a Tsallis distribution:

$$
W(p)=Z_{q}^{-1}\left[1-\beta(1-q) p^{2}\right]^{1 / 1-q},
$$

where $Z_{q}$ is, for $1<q<3$, the normalization factor. For $q \rightarrow 1$, the Tsallis distribution coincides with the MaxwellBoltzmann distribution, with Gaussian wings. For $q>1$, the Tsallis distribution displays instead power-law tails of the form $W(p) \sim 1 / p^{2 /(q-1)}$. The parameters $q$ and $\beta$, which characterize the Tsallis distribution, can be expressed in terms of the parameters of the optical potential. In particular, the $q$ parameter, which characterizes the nature of the tails of the distribution, is related to the depth of the optical lattice according to 


$$
q=1+44 \frac{E_{r}}{U_{0}}
$$

where $E_{r}$ is the atomic recoil energy [8].

The goal of the present work is to investigate experimentally whether the atomic momentum distribution is, indeed, a Tsallis distribution. However, as the semiclassical derivation of Ref. [11] involved spatial averaging and a classical description of the center-of-mass motion, we first verified to which extent these approximations affect the derived results by comparing them with full-quantum calculations. By using standard methods of quantum Monte Carlo simulations [12], we determined the atomic momentum distribution for an ensemble of atoms evolving in an optical lattice. The atomic motion is treated quantum mechanically, and the optical lattice is treated exactly (without spatial averaging). The coupling with the vacuum modes of the electromagnetic field is treated with the quantum jumps technique [12].

The results of our simulations are reported in Fig. 1. The data points in Fig. 1(a) are the results of our numerical calculations. The solid line is the best fit of the data points with a Tsallis function. The excellent agreement between the data points and the Tsallis distribution is evident, and it is quantitatively confirmed by an adjusted $R^{2}$ for the fit equal to 0.995 . We can conclude, therefore, that our quantum mechanical calculations confirm the results of Ref. [8] derived in the semiclassical limit: The atomic momentum distribution is a Tsallis function.

From our numerical simulations, we also determined, by fitting data as those in Fig. 1(a), the value of the $q$ parameter as a function of the depth $U_{0}$ of the optical potential, with results as in Fig. 1(b). These results validate the semiclassical prediction of Eq. (2) [dashed line in Fig. 1(b)] and motivate our experimental work.

Our experimental setup consists essentially of a threedimensional dissipative optical lattice of cesium atoms. A 3D configuration offers the significant advantage of confining the atoms in all directions, reducing in this way the loss of atoms from the optical lattice. The optical lattice is generated by four laser beams detuned of an amount $\Delta$ from the $F_{g}=4 \rightarrow F_{e}=5$ transition of the $D_{2}$ line and arranged in the so-called umbrellalike configuration [13]. One beam propagates in the $z$ direction; the three other beams propagate in the opposite direction, arranged along the edges of a triangular pyramid having the $z$ direction as an axis. This is the same lattice beams' geometry we used in our work on the ratchet effect [14], and we refer to that work for the details of the lattice beams' angles, relative amplitudes, and polarizations.

Cesium atoms are cooled and trapped in a magnetooptical trap, followed by a compression phase to reduce the spatial extent of the atomic cloud. The trap is then switched off, and after $15 \mathrm{~ms}$ of optical molasses the atoms are loaded in the optical lattice. The atoms are left to equilibrate in the optical lattice for $37 \mathrm{~ms}$. Then the optical lattice is switched off and the atomic cloud undergoes a
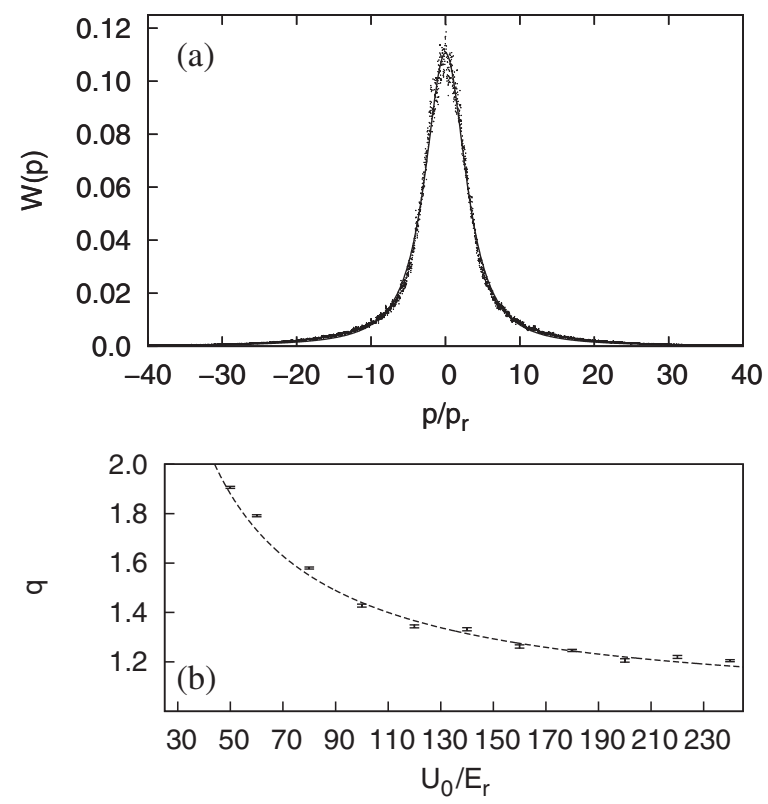

FIG. 1. (a) Results of quantum Monte Carlo simulations for the momentum distribution $W(p)$ of atoms cooled in a 1D lin $\perp$ lin optical lattice. The momentum $p$ is reported in units of the recoil momentum $p_{r}$. The data points correspond to the average of $n=10^{4}$ atomic trajectories. For each trajectory, the atom is initially in the internal state $|-\rangle$ and is in the ground state of a given well. The system then evolves in the lattice for a time $t_{f}=$ $100 / \omega_{r}$, where $\omega_{r}$ is the recoil frequency. The depth of the optical lattice is $U_{0}=60 E_{r}$, the detuning from atomic resonance is $\Delta=-20 \Gamma$, where $\Gamma$ is the width of the excited state. The line is the best fit of the data with the Tsallis distribution. The fit produced a $q$ value of $q=1.791 \pm 0.004$ with an adjusted $R^{2}$ equal to 0.995 . (b) Values for the $q$ parameter of the Tsallis distribution as a function of the depth of the optical potential. The data points correspond to our full-quantum Monte Carlo simulation and are obtained by fitting curves as those in (a) with a Tsallis distribution. The line represents the analytical result for $q$ as a function of $U_{0}$ derived in Ref. [8] in the semiclassical limit.

free expansion for $30 \mathrm{~ms}$. During the phase of free expansion, the atomic momentum distribution is mapped onto the atomic spatial distribution. After the phase of free expansion, the atomic cloud is illuminated by a retroreflected laser beam resonant with the $F_{g}=4 \rightarrow F_{e}=5$ transition for $1.5 \mathrm{~ms}$, and simultaneously an image of the cloud is taken using a charge-coupled device (CCD) camera. This image represents the momentum distribution of the atoms thermalized in the optical lattice. The CCD camera is oriented so as to image a plane which contains the $z$ axis. In this way, we can extract the momentum distribution along the $z$ direction from the images of the atomic cloud [15].

A typical example of our measurements of the atomic momentum distribution along the $z$ axis is shown in Fig. 2(a). That data set, as well as all data sets presented in this work, is obtained by averaging 200 images. Some remarks are in order to justify our procedure to analyze the data. Particular care has been taken to equilibrate the lattice 

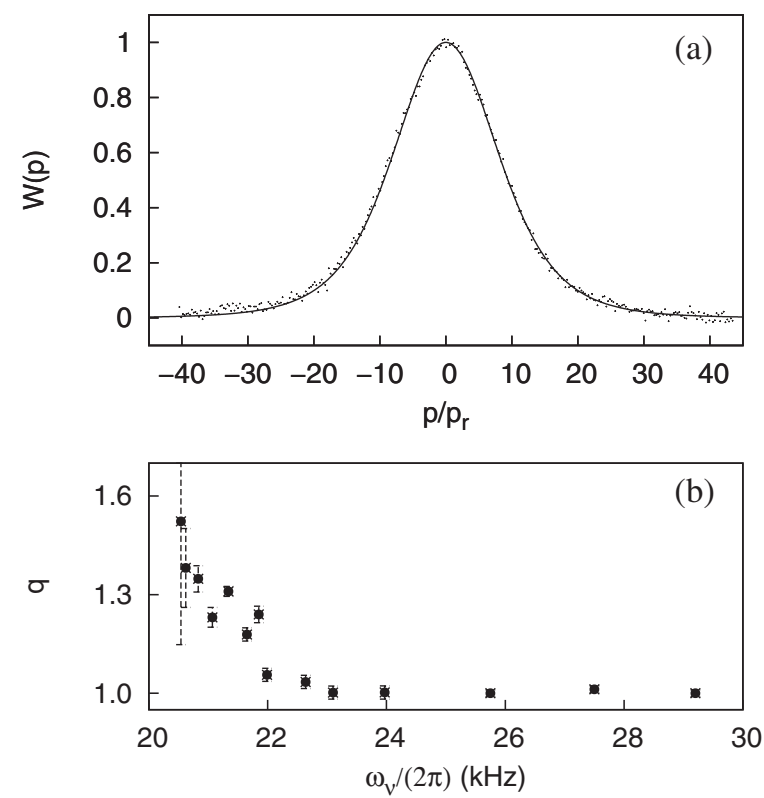

FIG. 2. (a) Experimental results for the momentum distribution of cold atoms in a 3D dissipative optical lattice (data points) and their best fit with a Tsallis function (solid line). The obtained $q$ value, equal to $1.310 \pm 0.015$, is derived by fitting only the right part of the momentum distribution. The adjusted $R^{2}$ is equal to 0.9985 . The parameters of the optical lattice are $\Delta=$ $-24 \Gamma, \omega_{\nu}=(2 \pi) 20.8 \mathrm{kHz}$. The distribution is normalized so that its maximum is equal to unity. (b) Values of the $q$ parameter as a function of the vibrational frequency at the bottom of the well, as obtained by fitting the experimental data with a Tsallis distribution.

beams, and this is reflected in the good symmetry of the results of our measurements; see Fig. 2(a). However, even a very small asymmetry may affect significantly the fitting procedure. This is particularly true for the analysis of the tail of the distribution, which requires one to identify the offset of the measurements very precisely.

To avoid introducing systematic errors in the data analysis due to a small unbalance of the lattice beams, we determined the parameters of the best fit with the Tsallis distribution by fitting only half of the curve. We arbitrarily chose the half with positive momentum, $p>0$ [16]. The fitting Tsallis distribution included also an offset, to take into account the unavoidable background present in the data. The result of this procedure is illustrated in Fig. 2(a): The solid line represents the best fit with a Tsallis function of the half side of the experimental data with $p>0$. In Fig. 2(a), the so-determined fitting function has been plotted also for $p<0$ to show that, because of the small asymmetry of the curve, the data points with $p<0$ have, in fact, a slightly different offset. This is precisely the reason which led us to restrict our fit to only one side of the measured momentum distribution.

We turn now to the quantitative analysis of the experimental data. Consider the data shown in Fig. 2(a). We fitted the side with $p>0$ of the momentum distribution with a Tsallis function. The agreement between the fit and the experimental data is excellent, as quantitatively characterized by an adjusted $R^{2}$ equal to 0.9985 . We can conclude, therefore, that the measured momentum distribution is a Tsallis distribution, in agreement with the theoretical predictions [8]. We made several measurements for different depths of the optical potential, which was varied by changing the lattice beams' intensity. The optical potential was characterized by measuring, via pump-probe spectroscopy [4], the vibrational frequency $\omega_{\nu}$ at the bottom of the wells, which scales as the square root of the potential depth. We notice that the conversion between intensity of the lattice beams and potential depth via pump-probe spectroscopy measurements introduces an error of the order of $1 \mathrm{kHz}$ on the offset of the frequency scale for our experiment [horizontal axis of Fig. 2(b)]. However, the potential depth was varied by changing the intensity of the lattice beams, which can be done with high accuracy. Therefore, although the absolute frequency has an error of $1 \mathrm{kHz}$, the error for the difference between two vibrational frequencies is much smaller, of the order of $1 \%$. The data for the vibrational frequency will, therefore, be reported with such an accuracy, under the assumption that only relative measurements are actually characterized by such an error. We explored the range of lattice depth for which we expect to observe the transition between Gaussian distributions $(q \rightarrow 1)$ and power-law tail distributions $(q>1)$. For all measurements, we found an excellent agreement between the fit with a Tsallis distribution and the experimental data. The adjusted $R^{2}$ was larger than 0.998 for all series of data except the one at the shallowest potential, for which the adjusted $R^{2}$ is equal to 0.988 , and the one at the deepest potential, for which we found an adjusted $R^{2}$ equal to 0.93 . From the fit of the experimental data, we derived the $q$ parameter for the Tsallis distribution as a function of the vibrational frequency, with results shown in Fig. 2(b). A direct comparison with our calculations [see Fig. 1(b)] is not possible because of the different dimensionality and atomic transition. However, we observe that the experimental findings agree qualitatively with the theoretical predictions: There is a transition between Gaussian distributions at deep potentials $\left(q \rightarrow 1\right.$ for large $\left.\omega_{\nu}\right)$ and non-Gaussian, power-law tail distributions at shallow potentials ( $q$ increasingly larger than 1 for decreasing $\omega_{\nu}$ ).

We have already stressed that the peculiar feature of the Tsallis distribution are the power-law tails. Previous studies of the momentum distribution of cold atoms in optical lattices were not conclusive about the nature of the tails of the momentum distribution [17]. The high value of the adjusted $R^{2}$ that we obtained for the fit of the momentum distributions of our experiment is a clear indication that the fitting Tsallis function well describes also the tails of the distribution. However, as this is an important point, we also analyzed separately the tails of the distribution to verify that they are consistent with the power-law tail distribution $W(p) \sim p^{2 /(1-q)}$. We refer to Fig. 3 for the analysis of the tails of the distribution. A data set is shown in Fig. 3(a), 

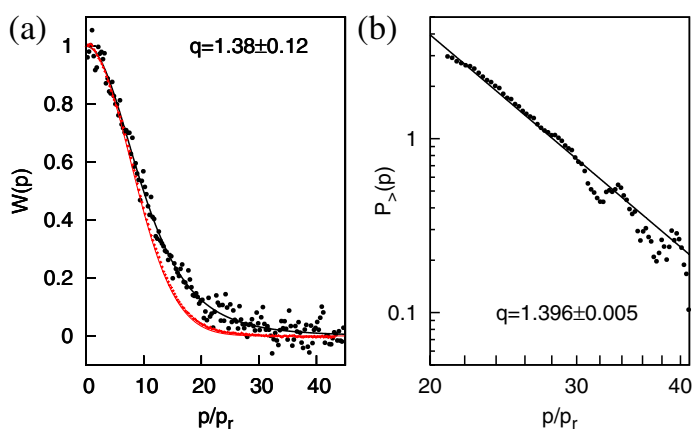

FIG. 3 (color online). (a) Experimental results for the atomic momentum distribution (black data points) and their best fit with a Tsallis function (black solid line). The value of the $q$ parameter derived from the fit is indicated in the figure. The adjusted $R^{2}$ is equal to 0.9985 . The parameters of the optical lattice are $\Delta=$ $-24 \Gamma, \omega_{\nu}=(2 \pi) 20.6 \mathrm{kHz}$. For comparison, the experimental data and relative fit for a deep optical potential $\left[\omega_{\nu}=\right.$ $(2 \pi) 27.5 \mathrm{kHz}$ ] are also reported (gray points and line, red online). The best fit with a Tsallis distribution produces $q=$ $1.01 \pm 0.01$; i.e., it is a Gaussian. (b) The data points are the experimental results for the distribution $P_{>}(p)$ [see Eq. (3)]. The solid line represents the best fit with the power law $c p^{(3-q) /(1-q)}$ of the data for $P_{>}(p)$ in the shown interval $p>20 p_{r}$.

together with the best fit with a Tsallis function. The fit produces a value for the $q$ parameter equal to $q=1.38 \pm$ 0.12 . Then we analyze just the tail of the distribution. As customary in the study of power-law tail distributions [18], we consider the (unnormalized) probability distribution

$$
P_{>}(p)=\int_{p}^{\infty} W(\tilde{p}) d \tilde{p} .
$$

Obviously, if $W(p)$ scales asymptotically as $p^{2 /(1-q)}$, we expect $P_{>}$to scale as $p^{(3-q) /(1-q)}$. We consider the tail of the distribution $P_{>}$and, more precisely, the portion of the data corresponding to a momentum $p>20 p_{r}$. As shown in the log-log plot in Fig. 3(b), the data for the tail of $P_{>}$are consistent with a power law, and a fit of these data with the function $c p^{(3-q) /(1-q)}$ produces a $q$ parameter equal to $q=$ $1.396 \pm 0.005$. This value, determined by fitting only the tail of the distribution, is equal, within the error, to the value for $q$ determined by fitting the entire distribution. This shows that the Tsallis distribution well describes the entire measured momentum distribution and constitutes direct evidence of the power-law nature of the tails of the measured distribution.

In conclusion, we demonstrated experimentally that the momentum distribution of cold atoms in dissipative optical lattices is a Tsallis distribution. The parameters of the distribution can be continuously varied by changing the lattice parameters. In particular, by changing the depth of the optical lattice, it is possible to change the momentum distribution from Gaussian at deep potentials to a powerlaw tail distribution at shallow potentials.

The implementation of tunable Tsallis distributions with cold atoms in dissipative optical lattices shows that this is a model system useful to study a variety of phenomena of statistical physics. In particular, the tunability of the Tsallis distribution will enable the experimental study of the correspondence between ergodicity breaking and power-law tail distributions, as recently established theoretically [10]. The use of tilted shallow optical lattices will also allow one to study anomalous transport in washboard potentials, a problem which has recently attracted much attention [19]. Finally, fluctuating nondissipative optical lattices could be used to investigate long tail distributions and rare events in quantum tunneling through fluctuating barriers [20].

We thank E. Lutz and G. Messin for very useful discussions. Financial support by EPSRC, United Kingdom and the Royal Society is acknowledged.

[1] For a review of Lévy distribution, see M. F. Shlesinger, G. M. Zaslavsky, and J. Klafter, Nature (London) 363, 31 (1993); M. F. Shlesinger, J. Klafter, and G. Zumofen, Am. J. Phys. 67, 1253 (1999).

[2] F. Bardou, J.-P. Bouchaud, A. Aspect, and C. CohenTannoudji, Lévy Statistics and Laser Cooling (Cambridge University Press, Cambridge, England, 2002).

[3] X. Brokmann, J. P. Hermier, G. Messin, P. Desbiolles, J.-P. Bouchaud, and M. Dahan, Phys. Rev. Lett. 90, 120601 (2003).

[4] For a recent review of optical lattices, see G. Grynberg and C. Mennerat-Robilliard, Phys. Rep. 355, 335 (2001).

[5] S. Marksteiner, K. Ellinger, and P. Zoller, Phys. Rev. A 53, 3409 (1996).

[6] W. Greenwood, P. Pax, and P. Meystre, Phys. Rev. A 56, 2109 (1997).

[7] H. Katori, S. Schlipf, and H. Walther, Phys. Rev. Lett. 79, 2221 (1997).

[8] E. Lutz, Phys. Rev. A 67, 051402(R) (2003).

[9] C. Tsallis, J. Stat. Phys. 52, 479 (1988).

[10] E. Lutz, Phys. Rev. Lett. 93, 190602 (2004).

[11] Y. Castin, J. Dalibard, and C. Cohen-Tannoudji, in Light Induced Kinetic Effects on Atoms, Ions and Molecules, edited by L. Moi et al. (ETS Editrice, Pisa, 1991).

[12] J. Dalibard, Y. Castin, and K. Mølmer, Phys. Rev. Lett. 68, 580 (1992).

[13] K. I. Petsas, A. B. Coates, and G. Grynberg, Phys. Rev. A 50, 5173 (1994).

[14] P. H. Jones, M. Goonasekera, and F. Renzoni, Phys. Rev. Lett. 93, 073904 (2004).

[15] We notice that the current setup also allows one to study anisotropic Tsallis distributions [see Y. Castin et al., Phys. Rev. A 50, 5092 (1994)], although this issue is not addressed in the present work.

[16] We verified that equivalent results for $q$ are obtained by fitting the $p<0$ side of the distribution.

[17] J. Jersblad, H. Ellmann, K. Støchkel, A. Kastberg, L. Sanchez-Palencia, and R. Kaiser, Phys. Rev. A 69, 013410 (2004).

[18] M. E. J. Newman, Contemp. Phys. 46, 323 (2005).

[19] I. Goychuk, H. Heinsalu, M. Patriarca, G. Schmid, and P. Hänggi, cond-mat/0509172.

[20] F. Bardou, Europhys. Lett. 39, 239 (1997). 